\title{
Paciente con trombocitopenia trombótica inducida por heparina en hemodiálisis: abordaje de la anticoagulación del circuito
}

\author{
Mirian García Martínezㄹ, Miguel Merino García², Alicia Labrador Pérez \\ ${ }^{1}$ Enfermera. Servicio de Nefrología. Hospital Universitario Marqués de Valdecilla. Santander. España \\ ${ }^{2}$ Enfermero. Servicio de Transfusiones. Complejo Asistencial Universitario de Burgos. España
}

\begin{abstract}
Resumen
El tratamiento renal sustitutivo de hemodiálisis es una técnica de depuración sanguínea extraterrenal, que requiere proteger al circuito extracorpóreo del paciente. Actualmente la heparina es el anticoagulante endovenoso de elección para evitar este tipo de complicaciones intradiálisis, siendo necesaria la individualización de las dosis por paciente.

La trombocitopenia trombótica inducida por heparina es uno de los posibles efectos secundarios producidos por la administración de heparina.

La bivalirudina es un inhibidor directo y específico de la trombina, útil en diferentes procedimientos, siendo utilizado en pacientes con problemas con la heparina y con fracaso renal crónico terminal que requieren terapias de diálisis continuas.
\end{abstract}

Caso clínico: Paciente de 46 años, con múltiples antecedentes personales. Al inicio de su proceso debutó con una Gangrena de Fournier secundaria a isquemia de extremidades inferiores, por lo que precisó tratamiento anticoagulante. Hasta la fecha no presentó alergias medicamentosas conocidas. Posteriormente se objetivó plaquetopenia progresiva con diagnóstico de trombocitopenia inducida por heparina tipo II por lo que se restringió la heparina de bajo peso molecular y la heparina sódica, indicándose anticoagulación con Sintrom y Bivalirudina en casos de procedimientos con alto riesgo de sangrado.

\section{Correspondencia: \\ Mirian García Martínez}

Servicio de Nefrología $1^{\text {a }}$ Planta, Pabellón 2 de Noviembre

Hospital Universitario Marqués de Valdecilla

Avda. Valdecilla, s/n. 39008 Santander

E-mail: mgm242@hotmail.com
Conclusiones: Los pacientes de hemodiálisis en nuestro hospital, tienen como pauta habitual de anticoagulación la heparina.

En este caso el diagnostico precoz de la trombocitopenia trombótica inducida por la heparina, fue crucial para evitar daños mayores, siendo el equipo de enfermería la piedra angular en el tratamiento en la sala de hemodiálisis.

PALABRAS CLAVE: trombocitopenia inducida químicamente; efectos adversos heparina; bivalirudina; hemodiálisis; trombosis.

Thrombotic patient with heparin-induced thrombocytopenia in hemodialysis: addressing circuit anticoagulation

\section{Introduction}

Renal replacement therapy for hemodialysis is an extrarenal blood clearance technique, which requires protecting the patient's extracorporeal circuit. Currently heparin is the gold-standard intravenous anticoagulant to avoid this type of intradialytic complications, being necessary the individualization of the doses per patient. Heparin-induced thrombotic thrombocytopenia is one of the possible side effects produced by the administration of heparin.

Bivalirudin is a direct and specific inhibitor of thrombin, useful in different procedures, being used in patients with problems with heparin and with terminal chronic renal failure requiring continuous dialysis therapies.

Clinical Report: Patient with 46 years old, with multiple personal history. At the beginning of his process, 
he debuted with a Fournier' gangrene secondary to ischemia of the lower extremities, for which he required anticoagulant treatment. To date, he has not had known drug allergies. Subsequently, progressive low platelet count with type II heparin-induced thrombocytopenia was diagnosed and low molecular weight heparin and sodium heparin were restricted. Anticoagulation with Sintrom and Bivalirudin was indicated in cases with high risk of bleeding.

Conclusions: Hemodialysis patients in our hospital have heparin as their usual anticoagulation regimen.

In this case, the early diagnosis of heparin-induced thrombotic thrombocytopenia was crucial to avoid major damage, with the nursing team being the cornerstone in the treatment in the hemodialysis unit.

KEYWORDS: thrombocytopenia/chemically induced; heparin/adverse effects; bivalirudin, hemodialysis, thrombosis.

\section{Introducción}

El tratamiento renal sustitutivo de hemodiálisis es una técnica de depuración sanguínea extraterrenal. Dicha técnica consiste en poner a circular la sangre del paciente por un circuito extracorpóreo, aumentando el riesgo de coagulación de la sangre. Para mantener el circuito permeable es necesario recurrir a fármacos anticoagulantes.

La adecuada anticoagulación en los pacientes con este tipo de tratamiento, es fundamental para prevenir las trombosis parciales del circuito extracorpóreo; provocando la disminución de la superficie de intercambio y la eficacia de la diálisis o las trombosis completas; imposibilitando la continuidad de la técnica.

Uno de los objetivos principales, se centra en utilizar la menor dosis de anticoagulante posible, para que el dializador y la cámara venosa, queden limpios de restos hemáticos, y que al finalizar la sesión se pueda hacer hemostasia de los sitios de punción del acceso vascular en un tiempo prudencial. Por otro lado, hay que tener en cuenta que una anticoagulación insuficiente produce pérdidas hemáticas y de hierro, y condiciona una disminución del rendimiento depurativo de la diálisis ${ }^{1,2}$.

La heparina sódica (HS) es actualmente el anticoagulante de primera elección, aunque su uso está siendo reemplazado por el empleo de la heparina de bajo peso molecular $(\mathrm{HBPM})^{3}$, no existiendo un acuerdo general para el empleo de uno u otro tipo de heparina ${ }^{4}$.

Otros aspectos importantes que se deben tener en cuenta son, el modo de actuación en situaciones especiales, como son la alergia/trombopenia inducida por heparina, y los pacientes con alto riesgo hemorrágico, así como la pauta de anticoagulación en aquellos pacientes que están recibiendo tratamiento con anticoagulantes orales.

La trombocitopenia inducida por heparina (TIH) es una complicación potencial del tratamiento con heparina, relativamente frecuente en la población general ${ }^{5}$.

Se han descrito dos formas: La primera se caracteriza por un descenso leve de las cifras de plaquetas que ocurre de 2 a 4 días después de iniciar la terapia. EI recuento plaquetario suele normalizarse sin necesidad de suspender el tratamiento. El mecanismo parece relacionarse con un efecto directo de la heparina sobre la activación plaquetaria. El mecanismo de producción es desconocido pero no es de naturaleza inmune, también se llama trombocitopenia inducida por heparina tipo I (TIH I).

La segunda forma se denomina trombocitopenia inducida por heparina tipo II (TIH II), es un desorden de carácter inmune, aparece en el $1 \%$ de los tratamientos con heparina, siendo ésta más intensa y progresiva. Se define como una caída del $50 \%$ de la cifra de plaquetas que ocurre entre 5 y 15 días de la primera exposición a la heparina y es uno de los posibles efectos secundarios producidos por su administración, provocando un riesgo importante morbimortalidad 6 .

Puede acompañarse de fenómenos trombóticos extensos tanto venosos como arteriales, con coagulación intravascular diseminada, mediado por anticuerpos contra el complejo heparina-factor 4 plaquetario (FP4) ${ }^{5-7}$.

La tasa de mortalidad de la TIH II y de las complicaciones trombo-embólicas asociadas se estima en 20$30 \%$ de los casos $^{8}$.

Entre las complicaciones tromboticas que aparecen con una frecuencia aumentada de 20 a 40 veces en relación a la población normal, cuyo riesgo persiste aún después de suspendida la heparina, cabe destacar: Infarto Agudo de Miocardio (IAM), coagulación intravascular diseminada (CID), embolismo pulmonar y ce- 
rebral, isquemia, necrosis cutánea y gangrena, siendo la manifestación más común la trombosis arterial de miembros inferiores?.

Como tratamiento anticoagulante alternativo a la heparina, en caso de las posibles complicaciones citadas anteriormente, en la paciente que posteriormente se expone se utilizó la bivalirudina.

La bivalirudina es un inhibidor directo y específico de la trombina, útil en procedimientos invasivos, quirúrgicos en fracaso multiorgánico, y en pacientes con fracaso renal crónico terminal que requieren terapias de diálisis continuas.

Los efectos anticoagulantes son visibles inmediatamente después de la inyección intravenosa (i.v.) y están relacionados con los niveles plasmáticos. El tiempo de coagulación retorna a la normalidad 1 hora después de la interrupción del tratamiento. Es eliminada del plasma por hidrólisis proteolítica y eliminación renal. La semi-vida de eliminación es de 25 minutos en los pacientes con la función renal normal y entre 34 y 57 minutos en los pacientes con insuficiencia renal moderada y severa, respectivamente. En los pacientes que dependen de la diálisis, la semi-vida de eliminación es de 3.5 horas. Aproximadamente el $25 \%$ de la dosis es aclarada por la hemodiálisis ${ }^{10}$.

\section{Caso Clínico}

Mujer de 46 años, con múltiples antecedentes personales. Se destaca una preclampsia en sus dos embarazos, siendo el primero en el año 2000 y repitiéndose en el 2002, diagnosticada de una hipertensión arterial (HTA) de difícil control, desde el año 2010, con hallazgo de adenoma e hiperplasia suprarrenal en el 2015.

El 29/09/2015 debutó con una Gangrena de Fournier secundaria a una isquemia de extremidades inferiores, precisando desbridaje quirúrgico, tratamiento en la cámara hiperbárica y realización de colostomía de descarga para evitar contaminación fecal (cerrada el 26/10/2016). Por ello la paciente precisó tratamiento anticoagulante con heparina. Hasta la fecha no presentó alergias medicamentosas conocidas.

El 08/10/2015 se objetivó plaquetopenia progresiva con diagnóstico de THI II por lo que se restringió la heparina de bajo peso molecular y la heparina sódica,
Tabla 1. Valoración inicial según los patrones funcionales de Marjory Gordon.
Patrón 1: Mantenimiento-Percepción de la Salud.

Conocedora de la situación, aunque no tiene claro su repercusión en su estado de salud y desconoce sus posibles

\section{Patrón 2: Nutricional y Metabóli-} co.

Dieta baja en potasio con ingesta de líquidos reducida. Presenta importantes edemas en cara y en miembros inferiores. Ganancia de peso interdiálisis de 3 kilogramos.

\section{Patrón 3: Eliminación.}

Patrón alterado por su enfermedad renal de base. Presenta disminución diuresis residual. Colostomía de descarga.

Patrón 4: Actividad - Ejercicio físico.

Dada su situación no realiza actividate.

\section{Patrón 5: Sueño - Descanso.}

No presenta problemas para conciliar el sueño. Refiere cansancio aumentado y necesidad de dormir más horas.

Patrón 6: Cognitivo- Perceptual.

Sin alteraciones complicaciones. des habituales de forma independienconcepto.

Refiere miedo por la situación actual y la repercusión que pueda tener el nuevo tratamiento, prefiere no pensar en ello. No es muy consciente de las posibles consecuencias.

\section{Patrón 8: Rol y Relaciones.}

Disminución actividad social y modificación del rol en la unidad familiar debido a su enfermedad. Vive con su marido y 2 hijos.

Por cuestiones laborales tanto el marido como los hijos colaboran como cuidadores y realiza las tareas del que por parte de la madre y hermanas no recibe casi ningún apoyo ni físico ni emocional.

Patrón 9: Sexualidad - Reproducción.

Sin alteraciones

Patrón 10: Adaptación y Tolerancia al estrés.

Refiere estar preocupada por la situación actual, intenta no pensar demasiado en todos los cambios que está teniendo.

Patrón 11: Valores - Creencias. Sin alteraciones.
Patrón 7: Autopercepción- Autohogar. Apoyo familiar disminuido, ya

indicándose anticoagulación con acenocumarol y bivalirudina en caso de procedimientos con alto riesgo de sangrado.

Durante este episodio comenzó con alteraciones renales siendo diagnosticada, de insuficiencia renal crónica avanzada por nefropatía isquémica, secundaria a trombosis arterial, en tratamiento renal sustitutivo desde 01/12/2015. La técnica pautada fue Hemodifiltración en línea pre dilucional con control volumétrico y sin heparina.

Precisó de la inserción de varios catéteres tunelizados en yugular, siendo las primeras sesiones poco satisfactorias por bajos rendimientos del catéter, con múltiples problemas de flujos y resistencias, aparte de coagulaciones repetidas del circuito debido a su restricción de heparina. Su tratamiento anticoagulante en ese momento correspondía únicamente a la toma de acenocumarol vía oral con sus respectivos controles y dosis 
Tabla 2. Plan de cuidados según taxonomía NANDA, NIC, NOC (Escala de medición: Nunca demostrado 1, Raramente demostrado 2, A veces demostrado 3, Frecuentemente demostrado 4, Siempre demostrado 5).

\begin{tabular}{|c|c|c|}
\hline $\begin{array}{l}\text { O0119 BAJA ATOESTIMA CRÓNICA } \\
\text { CARACTERÍSTICAS DEFINITORIAS: } \\
\text { La persona tiene expresiones negati- } \\
\text { vas sobre si misma. }\end{array}$ & $\begin{array}{l}\text { RESULTADOS NOC } \\
\text { 1205-Autoestima. Puntuación inicial: } 3 \\
\text { Puntuación diana: } 5 . \\
\text { Indicadores } \\
\text { 120501-Verbalizaciones de auto acep- } \\
\text { tación. Valor escala: } 4 \text {. } \\
\text { 120519-Sentimientos sobre su propia } \\
\text { persona. Valor escala: } 4 .\end{array}$ & $\begin{array}{l}\text { INTERVENCIONES NIC } \\
5400 \text {-Potenciación de la autoestima } \\
540003 \text { Animar a la paciente a identificar sus vir- } \\
\text { tudes. } \\
540018 \text { Facilitar un ambiente y actividades que } \\
\text { aumenten la autoestima. } \\
5270 \text {-Apoyo emocional } \\
527002 \text { Ayudar al paciente a que exprese los sen- } \\
\text { timientos de ansiedad. } \\
527011 \text { Permanecer con el paciente y proporcio- } \\
\text { nar sentimientos de seguridad durante los periodos } \\
\text { de más ansiedad. }\end{array}$ \\
\hline $\begin{array}{l}00146 \text { ANSIEDAD. } \\
\text { CARACTERÍSTICAS DEFINITORIAS: } \\
\text { Expresión preocupaciones debidas a } \\
\text { cambios en los acontecimientos vi- } \\
\text { tales. }\end{array}$ & $\begin{array}{l}\text { RESULTADO NOC } \\
\text { 1211-Nivel de ansiedad. Puntuación } \\
\text { inicial: } 3 \text { Puntuación diana: } 5 . \\
\text { Indicadores } \\
\text { 121117-Ansiedad verbalizada. Valor } \\
\text { Escala: } 4 \text {. } \\
\text { RESULTADO NOC } \\
\text { 1402-Autocontrol de la ansiedad Pun- } \\
\text { tuación inicial: } 3 \text { Puntuación diana: } 5 . \\
\text { Indicadores } \\
\text { 140215-Refiere ausencia de manifesta- } \\
\text { ciones físicas de ansiedad. Valor escala: } \\
4 . \\
\text { 140217-Controla respuesta ansiedad. } \\
\text { Valor escala } 4 .\end{array}$ & $\begin{array}{l}\text { INTERVENCIONES NIC } \\
\text { 4920-Escucha activa } \\
492011 \text { Favorecer expresión de sentimientos. } \\
492014 \text { Mostrar interés en el paciente. } \\
\text { 6160- Intervención en caso de crisis } \\
616002 \text { Ayudar en el desarrollo de nuevas habi- } \\
\text { lidades para solucionar problemas, si resulta ne- } \\
\text { cesario. }\end{array}$ \\
\hline $\begin{array}{l}00025 \text { - RIESGO DE DESEQUILIBRIO } \\
\text { DE VOLUMEN DE LÍQUIDOS } \\
\text { CARACTERÍSTICAS DEFINITORIAS: } \\
\text { Disminución de la diuresis. Entre } \\
\text { otras }\end{array}$ & $\begin{array}{l}\text { RESULTADO NOC } \\
\text { 0601-Equilibrio hídrico } \\
\text { Puntuación inicial: } 2 \text {. Puntuación diana: } \\
4 . \\
\text { Indicadores } \\
060116 \text { Hidratación cutánea. } \\
\text { Valor escala: } 4 \text {. }\end{array}$ & $\begin{array}{l}\text { INTERVENCIONES NIC } \\
2080 \text {-Manejo líquidos/electrolitos } \\
208016 \text { Llevar un registro preciso de ingesta y } \\
\text { eliminación. } \\
208021 \text { Observar si hay manifestaciones de des- } \\
\text { equilibrio de líquidos. } \\
4120 \text {-Manejo de líquidos } \\
412011 \text { Controlar los cambios de peso del pacien- } \\
\text { te antes y después de la diálisis, si corresponde. } \\
412023 \text { Pesar a diario y controlar la evolución. }\end{array}$ \\
\hline
\end{tabular}

personalizadas. El sellado del catéter se realizaba con uroquinasa según el protocolo de la unidad, siendo la dosis modificada durante el proceso, y las sesiones se realizaban utilizando bioconectores luer-lock.

Dada su situación en las sesiones de hemodiálisis, se planteó la utilización de bivalirudina como método extra. Precisó de numerosos ajustes de tratamiento, dado que se coagulaba el circuito antes de finalizar las sesiones, casi de continuo. En ese periodo de cambio de pauta, se alcanzó una dosis total de 70 miligramos intra-sesión. Una vez fijada ésta última pauta de tratamiento, desaparecieron las coagulaciones del circuito y el rendimiento del catéter fue mejorando, durante un periodo largo.

\section{Plan de cuidados de enfermería}

Primeramente se realizó una valoración integral de enfermería para la paciente según los patrones funcionales de Marjory Gordon (ver Tabla $\mathbf{1}$ ).

Tras realizarse la valoración inicial se identificaron varios diagnósticos enfermeros, destacando 3 de ellos. Para ello se utilizó la taxonomía NANDA, se obtuvieron los resultados NOC y sus indicadores de resultado, los cuales fueron valorados al inicio y al final de la situación que presentó la paciente en ese momento (ver Tabla 2).

Ajustada la dosis final del tratamiento anticoagulante extra, la paciente presentó una mejoría observable que duró a lo largo de los meses. A consecuencia de 
los constates cambios en su situación, la paciente continuó presentando problemas de salud, precisando nuevo estudio individualizado y siendo valorado un posible cambio en su tratamiento farmacológico. Esto puede ser motivo de nuevo caso clínico centrado en su nueva situación.

Con respecto al plan de cuidados individualizado, la intervención temprana en sus cambios emocionales, y la prestación de apoyo inmediato, mediante escucha activa, (entre otras actuaciones), proporcionadas en cada sesión de hemodiálisis, hizo posible una mejoría en su situación, reflejándose en los diferentes resultados obtenidos en las puntuaciones de la escala de medición superiores a las iniciales.

\section{Discusión y conclusiones}

La literatura refiere que la incidencia de TIH II es particularmente alta en pacientes con trasplante cardiaco, o sometidos a cirugía ortopédica, siendo infrecuente en pacientes pediátricos, obstétricos y en pacientes en programas de hemodiálisis ${ }^{11-14}$. También muestran que la incidencia de TIH II es hasta 10 veces mayor en los pacientes tratados con heparina intravenosa que en los pacientes tratados con heparina de bajo peso molecular ${ }^{15}$. En este caso el diagnostico precoz del TIH II por antecedentes personales de la paciente, el trabajo multidisciplinar realizado por parte de todos los profesionales y diferentes servicios; y la correcta individualización de los cuidados, fue crucial para evitar daños mayores, asegurando su correcto tratamiento de hemodiálisis hasta la fecha de estudio.

El trabajo enfermero fue esencial en el seguimiento y ajuste de las dosis de bivalidudina para evitar la continuidad de la coagulación del sistema, siendo el equipo de enfermería, la piedra angular en el tratamiento en la sala de hemodiálisis, dado que está presente en todo el procedimiento, manteniendo y facilitando la correcta individualización de los cuidados.

La elaboración de un plan de cuidados estandarizado, resultó un método eficaz en la práctica diaria, permitiendo la orientación de los mismos hacia objetivos comunes, mejorando la calidad asistencial de la paciente de manera individualizada.

El autor declara que no hay conflicto de interés.

\author{
Recibido: 13 diciembre 2017 \\ Revisado: 25 enero 2018 \\ Modificado: 28 febrero 2018 \\ Aceptado: 10 marzo 2018
}

\section{Bibliografía}

1. Fischer KG. Essentials of anticoagulation in hemodialysis. Hemodial Int. 2007;11:178-189.

2. Regnault $V$, de Maistre $E$, Carteaux JP, Gruel $Y$, Nguyen $P$, Tardy B et al. Platelet activation induced by human antibodies to interleukin-8. Blood. 2003; 101(4):1419-21.

3. Davenport A. What are the anticoagulation options for intermittent hemodialysis? Nat Rev Nephrol 2011 Jul; 7(9):499-508.

4. European Best Practice Guidelines Expert Group on Hemodialysis, European Renal Association: Section V. Chronic intermittent haemodialysis and prevention of clotting in the extracorporeal system. Nephrol Dial Transplant. 2002; 17 (Suppl 7):6371.

5. Aster RH. Heparin-induced thrombocytopenia and thrombosis. N Engl J Med. 1995; 332: 1374-6.

6. Davoren A, Aster RH. Heparin-induced thrombocytopenia and thrombosis. Am J Hematol. 2006; 81: 36-44.

7. Benítez M, González Gómez I, González Carmelo I, et al. Trombopenia Inmune inducida por heparina en hemodiálisis a propósito de un caso. Revisión de la literatura. Nefrologia. 2007; 27: 756-60.

8. Fischer KG. Essentials of anticoagulation in hemodiálysis. Hemodial Int. 2007; 11: 178-89.

9. Fort J, Piera L. Anticoagulación y fibrinolisis. En: Tratado de hemodiálisis. $2^{\circ}$ Edición. Jofre $R$, López Gómez JM, Luño J, Pérez García R, Rodríguez Benitez $\mathrm{P}$ (eds.). Barcelona: Editorial Médica Jims, S.L. ; 2006. pp: 201-11.

10. Eichler P, Lubenow N, Strobel U, Greinacher A. Antibodies against lepirudin are polyspecific and recognize epitopes on bivalirudin. Blood. 2004; 103(2):613-616. 
11. Hourigan LA, Walters DL, Keck SA, Dec GW. Heparin-induced thrombocytopenia: a common complication in cardiac transplant recipients. J Heart Lung Transplant. 2002; 21:1283-9.

12. Klenner AF, Lubenow N, Raschke R, Greinacher $A$. Heparin-induced thrombocytopenia in children: 12 new cases and review of the literature. Thromb Haemost. 2004; 91:719-24.

13. Fausett MB, Vogtlander $M$, Lee RM, Esplin MS, Branch DW, Rodgers GM, et al. Heparin-induced thrombocytopenia is rare in pregnancy. Am J Obstet Gynecol. 2001; 185:148-52.

14. 0 'Shea SI, Sands JJ, Nudo SA, Ortel TL. Frequency of anti-heparin-platelet factor 4 antibodies in hemodialysis patients and correlation with recurrent vascular access thrombosis. Am J Hematol. 2002; 69:72-3.

15. Martel N, Lee J, Wells PS. Risk for heparin-induced thrombocytopenia with unfractionated and low-molecular-weight heparin thromboprophylaxis: a meta-analysis. Blood. 2005; 106:2710-5.

Este artículo se distribuye bajo una Licencia Creative Commons Atribución-NoComercial 4.0 Internacional. https://creativecommons.org/licenses/by-nc/4.0/

\section{Open Access (c) (i) \&}

